Revista Complutense de Historia de América

ISSN: $1132-8312$

\title{
La Colombie sur la scène internationale durant l'entre-deux-guerres: motivations et enjeux
}

\author{
Christian Camilo Casas García ${ }^{1}$
}

Recibido: 4 de abril de 2018 / Aceptado: 27 de agosto de 2019

Résumé. L'entre-deux-guerres est une période où les échanges entre la Colombie et le reste du monde connurent un développement remarquable malgré l'état de subordination de sa diplomatie à l'égard de la politique étrangère des États-Unis, son principal partenaire économique. De ce fait, pour le gouvernement colombien, la lutte contre les impérialismes constitua un des piliers de sa politique étrangère, en appelant au renforcement de la coopération régionale et à la mobilisation de l'expertise internationale pour le développement matériel et humain des États latino-américains. Influencées par l'optimisme né à Versailles avec les idéaux wilsoniens, les différentes administrations qui se succédèrent durant cette période cherchèrent à caractériser la réalité régionale et renforcer les institutions étatiques pour la réalisation d'un projet de démocratie humaniste.

Mots-clés: Société internationale; petite puissance; coopération régionale; représentation, dépendance.

\section{[en] Colombia in the International Arena in the Interwar Period: Motives and Challenges}

\begin{abstract}
The inter-war period was a time when exchanges between Colombia and the rest of the world developed remarkably despite the subordination of the nation's diplomacy to the foreign policy of the United States, its main economic partner. As a result, for the Colombian government, the struggle against imperialism constituted one of the pillars of its foreign policy, calling for the strengthening of regional cooperation and the mobilization of international expertise for the material and human development of Latin American states. Influenced by the optimism born in Versailles and by Wilsonian ideals, the various succeeding administrations during this period sought to characterize regional realities and strengthen state institutions in favor of the materialization of a project of humanist democracy.
\end{abstract}

Keywords: International society; small powers; regional cooperation; representation; dependance.

Sumario. 1. Introduction. 2. L'accès de la Colombie au concert mondial. 3. La mise en place progressive d'une nouvelle politique étrangère. 4. Expertise technique et relations commerciales colombiennes: l'exemple des États totalitaires. 5. Le projet colombien d'une ligue des nations américaines: renforcer la coopération régionale. 6. Conclusion. 7. Bibliographie

Cómo citar: Casas Garcia Christian Camilo, N. (2020) La Colombie sur la scène internationale durant l'entre-deux-guerres: motivations et enjeux, en Revista Complutense de Historia de América 46, 197-218.

1 Doctorant en Histoire à l'École des Hautes Études en Sciences Sociales et chercheur associé au laboratoire d'études des Mondes Américains [France]

E-mail: christian.casasgarcia@ehess.fr 


\section{Introduction}

Depuis la fin du XIX ${ }^{\mathrm{e}}$ siècle, la Colombie est engagée dans un processus de coopération internationale multilatérale en vue du développement économique et social du pays. Après la fin des guerres civiles en 1903, ce processus s'est accéléré sous l'impulsion des présidents Marco Fidel Suárez (1918-1921) et Pedro Nel Ospina Vásquez (1922-1926), favorisant l'élaboration d'un important corps diplomatique colombien. Depuis les années 1920, ce pays jouissait d'une reconnaissance importante, étant l'un des seuls pays de l'Amérique latine bénéficiant d'une paix complète. Cette paix était garantie par le biais du respect des institutions libres, mais également par le biais de notables progrès dans les domaines économique, juridique ou encore institutionnel. La crise de 1929 a accéléré ce processus au lieu de le ralentir ${ }^{2}$. Stimulée par un énorme afflux d'investissements étrangers, la progression économique colombienne est en grande partie favorisée par les échanges extérieurs, permettant une croissance du PNB de $10 \%{ }^{3}$. Bien que la dépression économique mondiale de 1929 n'ait pu totalement épargner une économie désormais plus dépendante des marchés extérieurs, celle-ci reste l'une des rares à avoir continué sa course en avant dans l'Amérique du Sud. Ces circonstances ont contribué à augmenter son prestige sur la scène mondiale, comme en témoignent son entrée à la Société des Nations (désormais $\mathrm{SdN}$ ) et à l'Organisation internationale du travail (désormais OIT), et l'intérêt que la Colombie éveilla par son activité, ses richesses et son développement auprès des États plus industrialisés d'Europe.

Chacun de ces éléments contribua au développement des relations entre la Colombie et le monde durant l'entre-deux-guerres. Cependant, ce ne fut pas tant la place de ce pays dans le monde qui fut le moteur de ce développement, mais plutôt le fait qu'elle multiplia sans ambages ses relations avec les autres acteurs de la scène internationale. C'est un pays qui défendit sur la scène mondiale son attachement farouche à la souveraineté étatique et à son système démocratique, mais en postulant le concept "d'égalité entre les États".

Cette étude veut poser les bases d'une réflexion sur la contribution de l'entredeux-guerres au débat sur l'intégration et la place des "petits" États latino-américains dans le concert mondial, à travers l'exemple colombien ${ }^{4}$. C'est en ce sens, que nous envisageons présenter les principales orientations de la politique étrangère colombienne, ses motivations et ses enjeux durant l'entre-deux-guerres. La consultation des fonds d'archives du Bureau international du travail à Genève et les Archives Diplomatiques de La Courneuve, nous a apporté des éclairages importants concernant la politique étrangère de la Colombie. Même si les dépêches réalisées par les agents diplomatiques français sont avant tout le reflet des intérêts de la France en Amérique du Sud, les informations recueillies par ces diplomates constituent une base de documentation de première main pour mieux appréhender le rôle de ce pays aux niveaux régional et international. Quant aux archives du BIT, cette documentation a le mérite d'être riche en détails. Pour notre étude, nous avons principalement consulté les rapports réalisés par le correspondant de la

Gamboa Ardila, 2007 [2005]: 19.

Ibídem: 20 .

4 Débat repris après la Seconde Guerre mondiale dans le cadre des projets d'intégration régionale en Amérique latine, voire dans le mouvement des non-alignés. 
Colombie auprès du Bureau, Alfredo Vázquez Carrizosa (1909-2001), entre 1934 et 1942 .

Le gouvernement colombien centra ses efforts pour le renforcement de la coopération régionale, l'élargissement de son champ d'action au sein des institutions internationales genevoises et contribuer au positionnement des petites puissances latino-américaines en tant qu'acteurs "à haute moralité juridique". Pour la Colombie, le $\mathrm{XX}^{\mathrm{e}}$ siècle serait l'ère historique la plus importante pour l'Amérique latine, face à une Nord-Amérique et une Europe corrompues par leurs ambitions impérialistes. C'est à cette rencontre singulière de la Colombie avec le monde que cette contribution est consacrée.

\section{L'accès de la Colombie au concert mondial}

Jean-Claude Allain souligne que dès la fin du XIX ${ }^{\mathrm{e}}$ siècle, le nombre de petites puissances s'est considérablement accru. Il y avait 41 membres de la SdN en 1920, dont 15 États latino-américains. La Colombie en faisait partie. Le plus souvent, les petites puissances sont des États qui disposent de faibles moyens démographiques, économiques et de peu de moyens pour faire prévaloir leur volonté auprès des autres ${ }^{5}$. Cela mène les petits États à une situation durable de grande dépendance vis-à-vis des grandes puissances pour la sauvegarde de leurs intérêts vitaux et la reconnaissance de leur existence dans le concert mondial ${ }^{6}$. Tout ceci nous permet de construire sur la notion de puissance une réflexion plus ouverte intégrant les ressources naturelles et humaines, qui constituent pour l'Etat à la fois des atouts et des facteurs de contrainte. La Colombie ne présente pas les attributs d'une puissance. Sa population de 7.851.000 habitants et sa superficie de 1.148 .000 kilomètres carrés la positionnent au troisième rang parmi les États les plus étendus en superficie en Amérique du Sud7.

La Colombie au lendemain de la Grande Guerre est un pays inconnu. Cela est dû à l'isolement de la capitale dans les hautes terres de l'intérieur andin, on a parlé non sans exagération de la Colombie du XIX siècle comme le "Tibet de l'Amérique latine". À ce facteur d'éloignement avec le reste du monde, il faut ajouter que ce n'est pas un pays d'immigration. On compte quelques familles d'origine espagnole, française ou allemande au XIX ${ }^{\mathrm{e}}$ siècle, mais surtout des Syro-libanais et des Polonais d'origine juive plus nombreux entre 1930 et $1940^{\circ}$. Nous pensons que l'instabilité politique, la fragilité économique et son désordre juridique ont contribué aux faibles flux d'immigration. Néanmoins, le fait qui a marqué les relations complexes entre la Colombie et la société internationale au début du $\mathrm{XX}^{\mathrm{e}}$ siècle est la sécession de Panama en 1903.

\footnotetext{
Allain, 1986: 11-22.

Frank, 2012: 176-177.

D'après le service statistique de la SdN, les États les plus étendus en Amérique du Sud étaient les suivants: Brésil $(8.511 .000 \mathrm{~km})$; Pérou $(1.383 .000 \mathrm{~km})$; Colombie $(1.140 .000 \mathrm{~km})$ et Venezuela (1.020.000). En: Economic and Financial Section of the League of Nations, 1927: 167.

8 Gilhodes, 1996: 125.

9 Dépêche n 167 de M. Jean du Boisberranger, Chargé d'Affaires de la République française à Bogotá à M. Yvon Delbos, Député, Ministre des Affaires Étrangères à Paris, 20-XII-1937. Archives Diplomatiques de La Courneuve [France] (désormais ADC), Colombie, Série Correspondance Politique et Commerciale, $14 \mathrm{CPCOM}, \mathrm{N}^{\circ} 5$.
} 


\subsection{La Colombie et la Société des Nations}

Alors qu'elle vient de perdre le Panama, conséquence de la guerre des Mille Jours, la Colombie prit conscience entre 1918 et 1922 du caractère contre-productif de sa politique étrangère au sujet de la reconnaissance de cette province comme État indépendant ${ }^{10}$. Elle comprit en particulier le piège dans lequel elle risquait de tomber en essayant d'exclure ce pays de la SdN, s'érigeant par conséquent comme une menace pour la stabilité de la région américaine. Bogotá ne pouvait dévier du chemin emprunté dès sa participation à la Seconde Conférence de la Haye de 1907, pour se lancer dans une lutte qu'elle n'avait aucune chance de gagner pour récupérer ce territoire ${ }^{11}$. Les autorités colombiennes durent donc opter pour une autre stratégie afin de compenser cette perte, tout en accédant aux institutions genevoises après la reconnaissance de l'État panaméen. Les administrations qui se succédèrent n'avaient pas cessé de dénoncer cette affaire comme une "honte" et comme une "humiliation" 12. Les Colombiens considérèrent être les victimes des prétentions de Theodore Roosevelt (1901-1909) sur l'isthme, qui ornait encore l'écusson national ${ }^{13}$. Si la communauté internationale fut défavorable à sa position envers Panama, la Colombie accusa les États-Unis d'être une perpétuelle menace pour son indépendance réelle"14. "Nous pouvons être amis, sans que cette amitié favorise de leur part un désir de domination et d'entretenir chez nous une crainte de vasselage"15 déclara en 1931, le président Enrique Olaya Herrera (1930-1934). Ce fut ce sentiment de crainte qui la poussa à s'intégrer au nouveau système international issu en 1919, avec pour recours ultime l'invocation du Droit international et son intégration aux organismes internationaux de Genève.

Jane Rausch explique à ce propos que les États-Unis souhaitèrent rétablir une relation de confiance avec la Colombie en accordant des réparations matérielles, pour dissiper ainsi les ressentiments motivés par l'attitude arbitraire de certains gouvernements nord-américains ${ }^{16}$. Pour le délégué de ce pays auprès de la Conférence de la Paix, Antonio José Restrepo (1855-1933), l'aboutissement du projet de la SdN et de l'OIT permettrait aux républiques sud-américaines "de nous présenter le front haut devant ces tribunaux avec l'assurance d'être écoutés et que nos demandes soient favorablement accueillies"17. Nous pensons que la nature juridique du projet des organismes internationaux genevois marqua un tournant dans les relations diplomatiques des pays sud-américains avec le reste du monde, pour défendre leurs intérêts vis-à-vis des prétentions impérialistes des États-Unis. La perte du Panama, ainsi que les nombreuses occupations militaires nord-américaines en Amérique centrale éveillèrent parmi les élites politiques et économiques latino-américaines un ressentiment contre l'impérialisme étasunien et contre toute forme d'intrusion étrangère

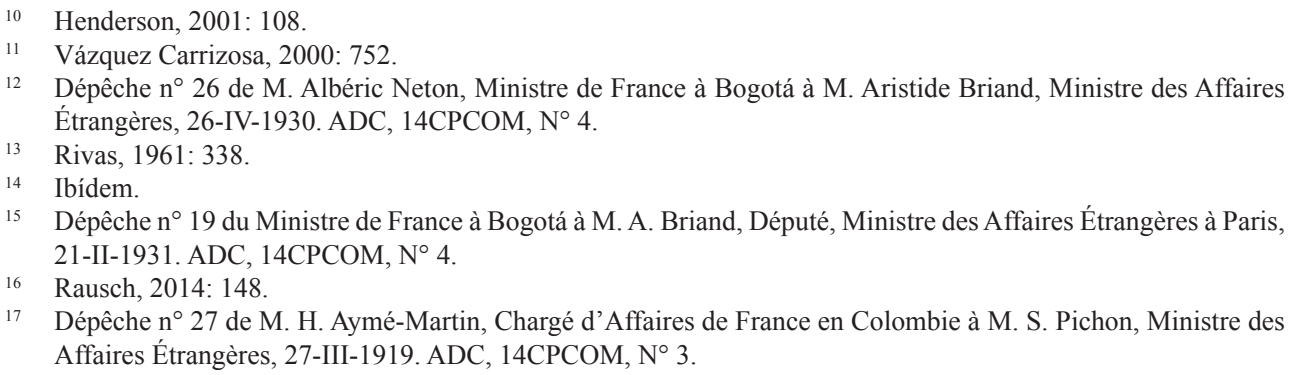

17 Dépêche n ${ }^{\circ} 27$ de M. H. Aymé-Martin, Chargé d'Affaires de France en Colombie à M. S. Pichon, Ministre des Affaires Étrangères, 27-III-1919. ADC, 14CPCOM, Nº 3 . 
dans leurs affaires internes ${ }^{18}$. Ceci constitua le premier facteur qui poussa les États de la région à intégrer massivement les institutions genevoises. Comme en témoigne l'importante demande de participations latino-américaines aux assemblées générales et pour la première fois, l'existence d'institutions qui voulurent protéger les petites puissances des dangereuses ambitions des grandes puissances.

Par une note datée du 9 février 1920, le juriste Henri Fromageot (1864-1949), fut consulté au sujet des réserves formulées par le gouvernement colombien concernant son adhésion à la $\mathrm{SdN}^{19}$. Il exposa qu'il n'y avait pas d'inconvénient pour que la Colombie adhère aux organismes genevois, sous la condition qu'elle s'engage à reconnaître Panama comme État indépendant, si le Congrès des États-Unis acceptait les clauses du Traité de $1914^{20}$. Le gouvernement souhaita bénéficier d'un soutien international pour faire pression au Congrès nord-américain pour la ratification de ce traité, et enfin obtenir l'indemnisation de 25 millions de dollars et compenser ainsi cette perte territoriale. Néanmoins, la demande d'adhésion colombienne parut assez singulière, si on considère qu'elle aboutit à ne pas reconnaître l'État panaméen, l'un des pays signataires du Traité de la SdN. L'accès de ce pays aux organes internationaux n'influença pas directement la ratification dudit traité en 1921, mais diversifia la politique étrangère colombienne et surtout attira l'attention des experts genevois. Pour les différentes administrations colombiennes durant l'entre-deux-guerres, l'expertise technique apportée par les institutions de Genève devait décentrer leurs liens de dépendance avec les États-Unis.

La Colombie fut admise à la SdN et à l'OIT le 3 mars 1920, en espérant de la part des États-Unis la construction de nouvelles relations, basées davantage sur la coopération économique et technique que sur la domination et l'inégalité. Les délégués colombiens à Genève promurent l'établissement d'une société internationale basée sur les principes d'égalité entre les États et le respect de la souveraineté. Tout cela fut utilisé par la Colombie pour positionner les petites puissances latino-américaines en tant qu'acteurs à "haute moralité juridique", à travers la progressive construction d'un projet de coopération américaine durant l'entre-deux-guerres, en s'inspirant du projet des ligues régionales présenté par le président uruguayen Baltasar Brum (1919-1923) en janvier 1923.

Le gouvernement colombien durant les années 1920 tenta de doter le pays d'une politique extérieure autonome, diversifiant et intensifiant tout à la fois ses relations avec les autres acteurs de la scène mondiale. Le gouvernement conservateur chercha très tôt une réforme du pacte de la $\mathrm{SdN}$, car les élites politiques colombiennes constatèrent que les projets discutés à Genève ne répondaient pas aux réalités régionales. Il chercha en outre, intégrer rapidement de changements législatifs permettant d'inclure les Conventions signées à la $\mathrm{SdN}$ et à l'OIT dans la loi positive du pays, mais la longue procédure parlementaire généralement employée en Colombie fut un obstacle pour l'adoption de ces mesures. À ce propos, M. Carrizosa explique lors de sa mission en Colombie en 1934, les difficultés rencontrées par le BIT pour faire avancer les discussions sur un Code du Travail, dont les débats dans les chambres avaient commencé depuis 1928. Il rapporte:

18 Occupations militaires étatsuniennes en Amérique centrale: Nicaragua (1912-1933), Haïti (1915-1934) et République Dominicaine (1916-1924).

19 Dépêche $\mathrm{n}^{\circ} 3$ de M. Langlais, Chargé d'Affaires de France en Colombie à M. Briand, Président du Conseil, Ministre des Affaires Étrangères, 21-V-1921. ADC, Série SdN, Colombie - dossiers géographiques, Nº 2037.

20 Vázquez Carrizosa, 2000: 14. 
La session du Congrès n'ayant lieu qu'une fois par an, nombreux sont les parlementaires qui présentent un, parfois deux ou trois projets de loi qui viennent s'ajouter aux initiatives du Gouvernement. De ce fait, il y a un tel encombrement lors des sessions parlementaires que les projets de loi sont condamnés chaque année à subir qu'une première discussion ou à ne pas être discutés du tout [...] Ajoutez à cela la disposition constitutionnelle de l'Article 97, et d'après laquelle les projets de loi qui n'auraient pas subi les trois débats parlementaires dans chacune des chambres ne seraient pas considérés que comme de simples projets de loi au cours de la session parlementaire suivante ${ }^{21}$.

\subsubsection{La question des particularités régionales et la menace communiste à Genève}

Depuis son accession, la Colombie participa à toutes les assemblées de la $\mathrm{SdN}$ avec un grand intérêt, malgré le manque de personnel technique qui lui faisait défaut. Ainsi, les délégués colombiens entre 1922 et 1933: Antonio José Restrepo et Francisco José Urrutia (1870-1950), avec les délégués d'autres pays de la région tels que l'Argentine, Panama, la République Dominicaine et le Chili s'investirent sur deux questions principales: l'urgence d'inclure les spécificités de la région latino-américaine aux débats internationaux et souligner la dangerosité des idées "communistes" et "socialistes" pour la communauté internationale.

En effet, l'adoption des mesures à Genève sur le plan social et économique ne prit pas en compte les diversités socio-économiques et culturelles des pays extra-européens. Lors de la première Conférence internationale du travail en 1919, le président Thomas Woodrow Wilson (1856-1924) et le délégué de l'Angleterre George Nicoll Barnes (1859-1940), remarquèrent que la Conférence devait discuter davantage le travail industriel et laisser de côté l'agriculture ${ }^{22}$. Pour les fonctionnaires permanents de l'OIT, il y avait un manque d'informations sur l'agriculture qui ne permettait pas de réaliser une convention sur le travail agraire ${ }^{23}$. D'après eux, l'agriculture différait des industries urbaines, et dans beaucoup de pays elle était entre les mains des petits propriétaires dans des conditions très variées. De plus, elle était conditionnée par les saisons et les besoins des familles concernées. Dans ce contexte, il était impossible d'analyser le travail agraire lors de cette conférence ${ }^{24}$. La Colombie étant un pays essentiellement agraire, souligna la nécessité de prendre en compte les spécificités locales des pays latino-américains, où l'industrie était généralement peu développée.

Pour Antonio José Restrepo: "biffer les particularités nationales de notre programme, c'est nous dire, à nous représentants des pays extra-européens: allez-vous en, puisque vous n'avez rien à faire ici (rires et applaudissements)" ${ }^{25}$. Le débat sur l'intégration des spécificités de la région latino-américaine aux assemblées et organes techniques genevois traversa toute la période de l'entre-deux-guerres, par une réforme du Pacte de la $\mathrm{SdN}$, et l'inefficacité des mesures prises à Genève ne fit qu'augmenter un sentiment de frustration parmi les délégations latino-américaines.

21 Report of A. Vazquez on his mission to Panama and Colombia, 13-IV-1934. Archives of the International Labour Office [Suisse] (désormais AILO), G900/89/3.

22 Conférence internationale du travail, 1919: 30.

23 Ibídem: 116.

24 Ibídem: 31.

25 Ibídem: 28 . 
Cela est à l'origine des projets régionaux concernant la création d'un institut interaméricain du travail et d'une ligue des nations américaines, en concurrence directe avec les travaux des organisations genevoises. Cette dernière fut présentée et formalisée par le président Alfonso López Pumarejo (1934-1938), et par le gouvernement de la République Dominicaine lors de la Conférence Panaméricaine de Buenos Aires de 1936. On reviendra plus en détail sur ce point.

Les élites politiques latino-américaines durant les années 1920 durent affronter un resurgissement des mouvements sociaux violents, qui furent rapidement qualifiés de "communistes" et de "socialistes", particulièrement en Colombie, où les grèves violentes connurent un essor important entre 1918 et $1929^{26}$. Bien que la menace des agents communistes ne fût pas imaginaire, elle semble avoir joué un rôle mineur dans la formation de grèves violentes, qui s'expliquent plutôt par le manque d'une législation structurée en matière sociale et du travail. Sur la scène genevoise, les délégués colombiens défendirent le projet d'exclure la Russie de la société internationale et de condamner sévèrement l'expansion des idées communistes dans les débats internationaux, malgré le rapprochement diplomatique entre la Russie et la Colombie dès 1928. Fabián Herrera León et Yannick Wehrli soulignent à juste titre que depuis la fondation des institutions internationales, Genève se pensa comme une réponse alternative à la planification soviétique, à travers un programme de régulation économique et sociale ${ }^{27}$. Mais côté sud-américain, Genève fut accusée de favoriser les idées communistes. Le délégué permanent de la Colombie auprès de l'OIT, Francisco José Urrutia, exposa devant la douzième session de la Conférence du travail en 1929, la vision de son gouvernement sur les idées bolcheviques et les raisons d'une exclusion de la Russie de la société internationale. Il déclara:

Le phénomène de la révolution russe a surpris tout le monde. Tout à coup, l'Empire des Tsars est tombé, et tombé dans la boue. Un autre gouvernement a pris sa place [...] nous ne savons pas exactement ce que se passe dans ce pays. Dans tous les cas, c'est une révolution sanglante et terrible qui est venue opprimer le pauvre peuple russe. [...] Il faut leur tenir tête, et au lieu de les accueillir et de les honorer de notre amitié, il faut les combattre [...] M. Lénine au moment de sa mort disait: "Messieurs, aussi longtemps que la révolution se limitera à la Russie, nous serons perdus. Il faut porter la révolution dans le monde entier". Voilà donc les Bolcheviques qui opèrent en Chine, où ils suscitent des révolutions, les voilà en Amérique du Sud où ils suscitent également des révolutions. Ils poursuivent leur activité en Bolivie, en Colombie, en Argentine, partout ${ }^{28}$.

Durant les années 1920, la Colombie souligna deux problèmes qui l'empêchèrent de nouer des relations stables avec les institutions genevoises: 1'absence d'un débat sur la réalité extra-européenne et le soupçon persistant que Genève était un vecteur des idées communistes. Cependant, ce fut à partir de l'arrivée au pouvoir du libéralisme en 1930, que les administrations colombiennes se chargèrent d'être en règle avec les normes promues depuis Genève concernant la coopération financière, l'instauration d'une politique sociale structurée et le renforcement des institutions

\footnotetext{
Sánchez, 1998: 105.

Herrera León - Wehrli, 2011: 156-167.

Conférence internationale du travail, 1929: 25.
} 
démocratiques. Si les institutions genevoises portèrent un vif intérêt à la Colombie, c'est parce qu'elles voulurent jouir d'une légitime réputation en Amérique latine, réputation obtenue à travers l'expertise technique notamment. Quant à la Colombie, les administrations qui se succédèrent eurent la volonté de former un gouvernement composé d'experts ${ }^{29}$.

\section{La mise en place progressive d'une nouvelle politique étrangère}

La politique étrangère colombienne connut une évolution incontestable depuis le début du $\mathrm{XX}^{\mathrm{e}}$ siècle. Nous avons évoqué dans la première partie de cette étude, ce qui constitue les principales causes de cette évolution. Rappelons qu'alors, les dirigeants colombiens, et ceci avant que Marco Fidel Suárez et Pedro Nel Ospina Vásquez augmentassent le nombre des agences consulaires en Europe et en Asie, eurent le sentiment de faire face à un problème de grande ampleur. L'anti-américanisme et l'opposition d'une partie de la population aux abus des étrangers suscitèrent des manifestations nationalistes et xénophobes que le gouvernement eut peine à contrôler. Cela alimenta sur le plan extérieur, en particulier aux États-Unis, une plus grande méfiance envers ce pays ${ }^{30}$. Les prétentions expansionnistes nord-américaines suscitèrent une grande méfiance parmi l'élite politique, mais sans parvenir à défendre leurs intérêts, car les États-Unis furent le principal partenaire économique de la Colombie, dépassant largement les investissements européens, qui après la fin de la Grande Guerre avaient considérablement diminué.

Alors que la relative modération du président Enrique Olaya Herrera à l'égard des autorités nord-américaines permit à ces derniers de pousser leurs avantages sur un certain nombre de questions, notamment le contrôle des finances et de l'industrie du pétrole, l'arrivée d'un gouvernement libéral au pouvoir en 1930 désireux de diversifier ses champs d'action dans le monde, annonça une période tendue pour les relations colombo-américaines. Les priorités internationales affichées par le président Herbert Hoover (1929-1933), en particulier son intention d'utiliser la pression économique et militaire en Amérique latine, risquaient d'alimenter la confrontation avec une Colombie de plus en plus ouvertement hostile à l'influence des États-Unis dans ses affaires intérieures, sans réussir à résister pour autant aux pressions économiques nord-américaines.

Cependant, la victoire électorale d'Alfonso López Pumarejo en 1934 annonça une nouvelle période pour les relations colombo-américaines, portées davantage sur la coopération et l'entente. Le nouveau président colombien annonça la mise en place du programme "la révolution en marche". Il voulut obtenir par des moyens pacifistes et constitutionnels, les mêmes résultats que l'on pouvait obtenir par des moyens violents. Cette période annonça pour le pays une modernisation importante de ses industries, de son économie et la conquête de divers marchés internationaux, notamment en Asie.

Entre 1930 et 1940, les autorités colombiennes adoptèrent une diplomatie qu'on pourrait qualifier "d'évitement des conflits". Il s'agit pour Bogotá d'introduire un

29 Safford, 1989: 21.

30 Dépêche n 65 de M. H. Aymé-Martin, Chargé d'Affaires de France en Colombie à M. Pichon, Ministre des Affaires Étrangères, 10-XI-1918. ADC, 14CPCOM, Nº 11. 
discours et des méthodes diplomatiques susceptibles de désarmer plus efficacement les craintes extérieures, qui empêchèrent le développement d'investissements étrangers et donc d'améliorer l'image et l'efficacité de sa politique étrangère. Parallèlement, à partir de 1934, cherchant à tirer profit de l'accroissement des communications qui se produisit après la Grande Guerre, le gouvernement colombien chercha de plus en plus à proposer des mesures "bénéfiques pour tous", pour la résolution des conflits frontaliers avec ses voisins notamment ${ }^{31}$.

\subsection{La Colombie et ses voisins}

Depuis les années 1930, la Colombie afficha une diplomatie orientée vers la construction d'une nouvelle image sur la scène internationale, et la construction d'une politique régionale latino-américaine pour faire face aux prétentions impérialistes des États-Unis et aux désordres en Europe. Durant cette période et malgré les conflits frontaliers avec le Pérou, le Brésil, l’Équateur, Panama, le Venezuela et le Nicaragua, Bogotá manifesta une volonté de rapprochement avec ses voisins latino-américains. Son but ne fut plus seulement de stabiliser ses relations au sein de son environnement latino-américain et principalement sud-américain ${ }^{32}$. Cet objectif demeura et se traduisit par une participation plus active de la Colombie aux mécanismes régionaux de coopération économique et de sécurité.

Depuis l'administration de Marco Fidel Suárez, qui formula un projet de coopération bolivarienne connu sous le nom de "doctrine Suárez", on retrouve le renforcement d'un projet avec l'objectif d'ériger la région latino-américaine comme la "gardienne du Droit international", à travers la coopération technique et juridique pour le renforcement des institutions démocratiques. En ce sens, l'Amérique latine représenta un laboratoire privilégié pour la mise en pratique d'un projet de coopération internationale basé sur des aspects tant "scientifiques" qu' "humanistes". Pour les élites politiques latino-américaines, la pacification du monde commença "chez soi”. Il était important pour la Colombie de mettre en place des liens privilégiés avec ses voisins, c'est-à-dire avec l'ensemble des pays limitrophes au sens large du terme: ses voisins continentaux et maritimes. L'établissement de bonnes relations au niveau régional constitua l'étape initiale d'un ambitieux programme pour faire de l'Amérique latine une région exemplaire dans la résolution pacifique des conflits internationaux.

En effet, l'insertion de la Colombie dans la communauté internationale se traduisit par la recherche d'une résolution pacifique d'un certain nombre de questions. La première fut sa relation avec ses voisins $\mathrm{s}^{33}$. Les proclamations de solidarité avec les évocations au Libérateur commun Simón Bolívar se succédèrent, mais la réalité fut celle d'une profonde méfiance de part et d'autre. En 1928, la Colombie reconnut la souveraineté de la République du Nicaragua sur la côte des Mosquitos, comprise entre le Cap de Gracias a Dios et le fleuve San Juan. La Colombie avait depuis le $\mathrm{XIX}^{\mathrm{e}}$ siècle revendiqué cette côte. Les îles Mangles furent également attribuées au Nicaragua. En échange, la juridiction et la souveraineté territoriale furent reconnues

\footnotetext{
Henderson, 2001: 224.

Castillo, 1956: 218.

Rivas, 1961: 685.
} 
à la Colombie sur les îles San Andrés et Providencia, ce qui rétablit les bonnes relations entre les deux pays.

En revanche, la Colombie eut un conflit sérieux avec le Venezuela qui revendiquait la possession de la boucle autour de San Faustino. Le Venezuela refusa la délimitation proposée par la sentence arbitrale de la reine d'Espagne María Cristina du 16 mars 1891, entre l'Arauca et le Meta et la ligne de frontière au nord et à l'ouest de Yavita et de Pimchin. Le Venezuela refusa de reconnaître l'arbitrage espagnol et les deux États firent appel à l'expertise technique suisse en novembre 1916, pour conclure la délimitation définitive de l'ensemble de leur frontière. La sentence arbitrale de la Suisse fut rendue le 24 mars 1922, prévoyant l'exécution partielle de la délimitation antérieure et l'envoi d'une commission pour la délimitation du tracé de la frontière entre les deux pays ${ }^{34}$. Ce conflit eut des répercussions sérieuses et empoisonna les relations entre Bogotá et Caracas. Ce n'est que par le Traité López de Mesa-Gil Borges d'avril 1941, au travers duquel la Colombie et le Venezuela reconnurent que cette délimitation frontalière serait définitive, que les relations entre les deux pays s'approfondirent. Le gouvernement colombien, le milieu des affaires et les intellectuels prirent conscience que la coopération entre les deux pays était une nécessité avec des avantages communs. Par-delà les conflits territoriaux, des efforts ont été faits pour augmenter les échanges colombo-vénézuéliens. En particulier les échanges commerciaux, les investissements croisés et les échanges culturels visèrent à ressouder les liens historiques qui unissaient les deux nations, capables de résister face aux difficultés ponctuelles.

Avec le Pérou, la guerre livrée en Amazonie pour le port de Leticia en 1932 laissa des traces qui se traduisirent par une certaine méfiance et des relations limitées sur le plan diplomatique et économique ${ }^{35}$. La résolution de ce conflit fut présentée comme la plus grande victoire du système de la sécurité collective. Mais en réalité, ce fut grâce aux commissions régionales que le conflit de Leticia trouva une solution effective. Cela nourrit une réflexion parmi les élites politiques colombiennes, et plus largement latino-américaines, sur l'efficacité de la $\mathrm{SdN}$ et la nécessité de créer une institution régionale pour débattre entre elles d'affaires concernant l'Amérique latine.

\subsubsection{L'élargissement de la politique extérieure}

Pour favoriser le développement interne, le gouvernement libéral entreprit une nouvelle réorientation diplomatique, en établissant des liens plus solides avec les autres acteurs de la scène internationale. Cette réorientation fut favorisée par l'expérience internationale des présidents Enrique Olaya Herrera ${ }^{36}$, Alfonso López Pumarejo ${ }^{37}$

34 Dépêche n 45 de M. Langlais, Chargé d'Affaires de la République française en Colombie à M. Poincaré, Président du Conseil, Ministre des Affaires Étrangères, 29-XII-1922. ADC, 14CPCOM, № 3.

35 Dépêche n 174 du Ministre de France à Bogotá à M. Pierre Laval, Sénateur, Président du Conseil, Ministre des Affaires Étrangères, 7-IX-1935. ADC, 14CPCOM, N 4.

36 Enrique Olaya Herrera, après avoir fait ses études de droit à l’Université Républicaine de Bogotá, accéda à 1’Université Libre de Bruxelles pour réaliser des études en diplomatie et en sociologie en 1906. Dès son retour en Colombie, il commença son cursus honorum au sein du Parti Libéral en 1909. Grâce au soutien qu'il apporta à la campagne présidentielle de Carlos Eugenio Restrepo (1910-1914), Olaya fut nommé ministre des Relations Extérieures en 1910, puis, en 1921, sous l'administration de Jorge Holguín (1921-1922). Enfin, entre 1922 et 1929, il occupa le poste de ministre plénipotentiaire de Colombie à Washington.

37 Alfonso López Pumarejo fit ses études universitaires en finances au London School of Economics and Political Science, puis aux États-Unis, où il poursuivit ses études en économie et commerce à la Packard School de New 
et Eduardo Santos (1938-1942) $)^{38}$ et par la volonté de professionnaliser leurs agents diplomatiques et consulaires. Il en résulte un dynamisme qui plaça la République de Colombie comme un membre sud-américain actif pour la protection du Droit international, et pour la prise en compte des particularités latino-américaines par rapport à l'Europe et aux États-Unis ${ }^{39}$.

C'est une politique à directions multiples qui tendait à stabiliser la coopération entre la Colombie et les différents acteurs de la scène mondiale, notamment avec les États-Unis. La Colombie chercha à nouer des relations moins conflictuelles et plus respectueuses concernant la souveraineté de l'État ${ }^{40}$. Ainsi, Bogotá tenta d'établir des liens socio-économiques et politiques plus solides avec Washington. Pour le président nord-américain Franklin Delano Roosevelt (1933-1945), et pour son secrétaire d'État Cordell Hull (1933-1944), l'inauguration d'une nouvelle ère dans les relations entre la Grande République du Nord et l'Amérique hispanique fut essentielle. La politique de bon voisinage (Good Neighbor Policy) lancée par cette administration, modifia radicalement l'atmosphère d'inquiétude dans laquelle évoluèrent leurs relations diplomatiques. Pendant de longues années, ces relations furent menacées par "le danger d'intervention et de restrictions apportées à la souveraineté nationale d'un des États de l'Union Panaméricaine"41. Si durant les années 1920 le discours antiimpérialiste fut caractéristique dans tous les pays latino-américains, principalement caribéens, la politique de bon voisinage réussit à dissiper les anciennes tensions et les États-Unis établirent une influence croissante, sur une base de confiance et d'amitié. L'impérialisme continua à être une menace pour le gouvernement libéral d'Alfonso López Pumarejo, mais plutôt centrée sur les États totalitaires, en ayant une attitude méprisante à l'égard de l'Europe, qui devint courante dans les discours politiques et dans la presse officielle. La légitimité des institutions genevoises fut contestée davantage et une reformulation de la coopération régionale s'imposa dans les agendas des chancelleries latino-américaines et dans les conférences panaméricaines.

Après 1936, la Colombie réexamina sa position à l'égard des États-Unis, en exigeant davantage de garanties sur le plan économique, en échange d'un appui inconditionnel à la politique extérieure nord-américaine. Le rapprochement de la Colombie et des États-Unis fut d'une très grande utilité pour les Colombiens. Par exemple, la visite du National Research Council en mars 1941 aida à réorienter les projets industriels en temps de guerre, en limitant les importations et favorisant la création de nouvelles industries au niveau local pour remplacer les marchandises provenant de l'étranger, comme le montre l'usine de sodas inaugurée à Zipaquirá au cours de l'année $1941^{42}$. Précisément, pour l'étude des relations

\footnotetext{
York. Sa formation, lui permit de créer des liens forts avec quelques groupes financiers en Amérique du Nord. Il coordonna la campagne présidentielle d'Olaya Herrera en 1930, lui permettant d'acquérir une certaine expérience diplomatique. Il participa par exemple à la négociation du Traité de paix entre la Colombie et le Pérou, qui eut lieu à Rio de Janeiro en 1933, dont il joua le rôle de médiateur.

38 Eduardo Santos entama ses études supérieures en France, puis il fut nommé de 1910 à 1914 secrétaire du Consul de Colombie à Paris. Durant l'administration d'Enrique Olaya Herrera, Santos fut nommé ministre des Relations Extérieures. Finalement, sous l'administration de López Pumarejo, il intégra la délégation colombienne à la SdN de 1934 à 1938.

39 Urrutia, 1920: 270.

40 Gilhodes, 1996: 127.

41 Dépêche n 265 de M. Jules Henry, Chargé d'Affaires de France aux États-Unis à M. Louis Barthou, Ministre des Affaires Étrangères, 29-VI-1934. ADC, 14CPCOM, $\mathrm{N}^{\circ} 4$.

42 Monthly reports on Colombia, 15-XI-1938. AILO, P2589.
} 
colombo-américaines durant l'entre-deux-guerres la question est la suivante: est-ce que l'économie et la politique colombienne furent complètement dominées par les Etats-Unis ? Plus qu'auparavant, le gouvernement de la Colombie fut subordonné à la politique extérieure des Nord-américains. Si le positionnement stratégique de la Colombie dans la zone Caraïbe ne se renforça pas par sa présence à la $\mathrm{SdN}$, il fallait tirer les avantages de cette situation. La croissante dépendance économique à l'égard des États-Unis favorisa l'exportation de son café, le maintien d'une économie de marché face à la crise du continent européen et le renforcement de 1'oligarchie bipartite ${ }^{43}$.

La Colombie chercha aussi à explorer les contacts possibles avec le reste du monde avant 1940. Pour cela, elle resserra ses liens avec les pays du continent européen. Le développement des échanges entre ce pays et les États européens fut indéniable, surtout depuis la fin du XIX ${ }^{\mathrm{e}}$ siècle ; stimulé par un essor sans précédent des échanges commerciaux et par la mise en place d'un partenariat de développement dans les domaines de la salubrité ; l'éducation ; les finances ; l'industrie, ainsi que la modernisation des forces policières et militaires. L'approche européenne ne fut pas conjoncturelle, elle répondit à des profondes relations avec certaines puissances européennes. Alors que le pays vit une transformation radicale de ses structures sociopolitiques et économiques, les pays européens tels que la France ou l'Allemagne offrirent à la République colombienne une coopération importante pour la gestion de ses importantes ressources naturelles et la modernisation de ses systèmes éducatif, militaire et judiciaire. Le domaine des échanges commerciaux permit de concevoir des relations stables et croissantes. Si le pourcentage des exportations colombiennes entre 1920 et 1940 se dirigeant vers l'Europe semblait décroître, résultat de la prédominance des États-Unis sur l'économie colombienne et des difficultés du contexte de la crise de 1930, les exportations colombiennes d'émeraudes et de platine vers le continent européen continuèrent sa marche ${ }^{44}$. Ceci étant pris en considération, l'Europe ne fut qu'un élément de diversification de la politique étrangère colombienne. En aucun cas, les relations de ce pays avec les États européens ne pouvaient se substituer à ses relations avec les États-Unis.

L'entre-deux-guerres incita la Colombie à la recherche de nouveaux partenaires dans le domaine économique en Asie. Les importations japonaises en Colombie, que la Grande Guerre inaugura, ne firent que s'accentuer au cours des années 1920 et 1930. Dès 1919, plusieurs magasins dans les principales villes du pays enregistrèrent la réception d'un important nombre d'objets nippons qui furent acheminés en Colombie par la route du Pacifique (via Panama-Buenaventura) principalement des jouets, des porcelaines et des bibelots ${ }^{45}$. À défaut de représentation diplomatique, le gouvernement nippon nomma vers le milieu de l'année 1919 un consul honoraire à Bogotá. Le gouvernement colombien en réponse, envoya à Yokohama un consul général, Eduardo Espinoza Guzmán, qui exerçait à La Havane les mêmes fonctions. Mais ce ne fut qu'en 1930 que Tokyo établit une légation officielle en Colombie, décision motivée par la volonté d'encourager le commerce japonais et d'élargir le cercle à leurs fournisseurs et clients en Amérique du Sud. Par ailleurs, le Japon vou-

43 Gamboa Ardila, 2007 [2005]: 32.

44 Ibídem: 88

45 Dépêche n 102 de M. H. Aymé-Martin, Chargé d'Affaires de France en Colombie à M. S. Pichon, Ministre des Affaires Étrangères, 31-XII-1919. ADC, 14CPCOM, Nº 3. 
lut profiter des facilités que lui offrit ce pays, ayant besoin d'équipements industriels et d'une main-d'œuvre abondante ${ }^{46}$.

Pour Bogotá, le développement du pays devait se caractériser par la défense des pratiques démocratiques, l'État de droit, l'égalité, la justice et le développement de l'économie. Parallèlement, le concept d'une société plus unie se mit peu à peu au service de la diplomatie colombienne, en particulier pour le développement des missions d'expertise, élément fondamental de la politique étrangère de la Colombie de l'entre-deux-guerres. L'expertise technique étant un élément important dans l'intégration de ce pays dans les affaires d'intérêt commun, mérite une analyse approfondie dans notre étude. Cette expertise offrit aux petites puissances latinoaméricaines la possibilité de se faire remarquer dans le concert mondial et d'intégrer les projets réalisés par la société internationale. Nous pensons que cet aspect joua un rôle déterminant dans les relations de la Colombie avec le reste du monde.

\section{Expertise technique et relations commerciales colombiennes: l'exemple des États totalitaires}

À côté du pouvoir grandissant des Nord-Américains, qui atteignit son apogée au cours des années 1920, grâce à la mission Kemmerer de 1922, grandit aussi l'influence allemande et japonaise. Depuis 1933, l'Amérique du Sud se heurta à la concurrence technique de nouveaux venus, aux efforts combinés de l'Allemagne et du Japon. Dans tous les pays sud-américains, les puissances totalitaires s'appuyèrent sur un important nombre de nationaux dans la région; elles profitèrent de la complémentarité de l'économie sud-américaine avec l'économie des États-Unis et elles séduisirent les élites politiques traditionnelles avec leurs discours sur la parenté des races. Enfin, elles mobilisèrent un discours politique qui convenait mieux aux conservateurs que le discours de la solidarité américaine promu par Cordell Hull ${ }^{47}$.

L'élite politique colombienne s'intéressa à la coopération technique de l'Allemagne et du Japon afin de combler le manque de personnel qualifié. Le manque d'experts en statistiques, finances et questions sociales fut un obstacle pour connaître les spécificités de chaque région du pays. Ces experts venus de l'Europe et du Japon assuraient le développement interne, la modernisation du pays pour conquérir de nouveaux marchés, l'arrivée de nombreux capitaux étrangers et l'appropriation de nouvelles techniques de production. Les grandes puissances tirèrent profit de cette expertise pour avoir un contrôle dans l'exploitation des ressources naturelles abondantes, bénéficièrent des avantages commerciaux et fiscaux et accrurent progressivement leur influence dans la région. L'expertise technique constitua pour l'Allemagne et le Japon un facteur de soft power dans leurs relations avec la Colombie, afin d'augmenter une influence sur le plan économique, commercial et financier avec des avantages communs.

Entre la Colombie et les États totalitaires, des échanges directs et actifs eurent lieu. Cuivre, pétrole, café, viande, coton, etc., produits dont la Colombie regorgeait

46 Dépêche n 75 de M. D. de Martel, Ambassadeur de la République française au Japon à M. le Ministre des Affaires Étrangères, 26-VII-1930. ADC, 14CPCOM, N²2.

47 Dépêche n ${ }^{\circ} 272$ de l'Ambassadeur de France aux États-Unis à M. Georges Bonnet, Ministre des Affaires Étrangères, 28-III-1928. ADC, Série SdN, N 796. 
furent recherchés par l'Allemagne et le Japon, auxquels ils faisaient défaut. Entre les régimes totalitaires d'Europe et d'Asie et la Colombie le troc devient la règle. Les États-Unis ne pouvaient, au contraire, trouver profit que dans un système commercial triangulaire, incapables d'acheter suffisamment de matières premières aux nations sud-américaines ${ }^{48}$.

L'Amérique du Sud est souvent citée par l'historiographie comme l'exemple caractéristique du dumping japonais ${ }^{49}$. Depuis 1923, tissus de soie, de coton, appareils photographiques, jouets, armes, articles de sport, appareils électriques, boutons, crayons, brosses à dents, bijouterie, parfums, faïences, verreries, celluloïd, tapis ou laques arrivèrent de Yokohama et de Nagasaki pour inonder le marché sud-américain. Les industriels japonais envoyèrent des missions d'expertise afin de mieux connâ̂tre la réalité de chaque pays et mieux s'adapter aux besoins des populations. C'est pour cela que l'empereur Hirohito (1926-1989) et ses ministres décidèrent d'envoyer une mission commerciale japonaise en Amérique du Sud en 1927, en vue d'étudier les possibilités d'expansion commerciale que leur offrit le marché sud-américain et accroître ainsi leur influence dans une région inconnue pour la majorité des Japonais. En 1927, l'Empire du Soleil Levant vendit à Barranquilla pour 200.000 yens d'investissement : 1.600 soieries, 3.000 porcelaines et poteries, ainsi que 4.000 objets en celluloïd. À Cali à la même période, les commerçants nippons vendirent pour la même somme 600 objets d'art et 2.400 cotonnades $^{50}$.

En 1928, le diplomate Takemaro Kobayashi fut envoyé en Colombie en tant que chef d'une mission commerciale par le Ministère des Affaires Étrangères du Japon, accompagné par Bunsaku Mori (1890-1938), expert commercial et représentant de 1'Association des fabriques de faïence et de porcelaine du Japon ${ }^{51}$. Cette mission commerciale débarqua début février à Buenaventura, sur le Pacifique et se rendit à Cali, ville en plein développement depuis 1910, grâce en partie par le chemin de fer du Pacifique qui la connecta à un réseau économique plus vaste. Ces experts nippons visitèrent également Barranquilla, les vallées de Cundinamarca, le Cauca et les principales villes placées à côté du fleuve Magdalena. Arrivés à Bogotá, le 29 février, Takemaro Kobayashi fut accueilli par le Théâtre Municipal pour donner une conférence en espagnol devant un nombreux public. À cet évènement assista le président de la République, les ministres des Industries et des Relations Extérieures et d'autres personnalités, telle que Sanín Cano (1861-1957), notable écrivain colombien. S'exprimant en "correct espagnol" 52 , le chef de la mission fit un discours exposant les débuts des relations intellectuelles et économiques entre l'Empire du Soleil Levant et le reste du monde. Il insista notamment sur le progrès de l'éducation primaire, ainsi que sur le développement de l'enseignement technique et artistique que devait unir les deux pays. Un extrait du journal El Tiempo daté du 2 mars 1928 rapporte: “en ce qui concerne les relations spirituelles, l'orateur a manifesté qu'il existe entre la Colombie et le Japon un courant salutaire de sympathie et de compréhension dans les heures de joie et de triomphe, ainsi que dans les heures de douleur mutue-

\footnotetext{
48 Randall, 1992: 111.

49 Presseisen, 2013: 23.

50 Dépêche $\mathrm{n}^{\circ} 130$ de M. Méric de Bellefon, Consul de France à Yokohama à M. le Ministre des Affaires Étrangères à Paris, 13-VIII-1927. ADC, Série E Asie, Japon, 39CPCOM, Nº 79.

51 Dépêche $\mathrm{n}^{\circ} 38$ du Ministre de la République en Colombie à M. le Ministre des Affaires Étrangères, 2-III-1927. ADC, 39CPCOM, N 79 .

52 Ibídem.
} 
lles, et en raison de cela, les Japonais voyaient en Colombie un pays de leur prédilection spéciale" 53 . Cette conférence fut suivie par une série de projections cinématographiques, les unes de caractère purement technique et statistique sur l'économie japonaise, les autres plus pittoresques, furent consacrées à montrer la ville de Tokyo, son université et la gare centrale, ainsi que le double pont du palais impérial et les atouts économiques d'autres villes comme Kyoto, Osaka, Nagoya et Nara. D'autres extraits cinématographiques furent dédiés aux méthodes de fabrication du celluloïd, des soieries, de la bière, du papier en pâte de bois et surtout la célèbre usine flottante pour les conserves de crabes, unique à cette époque ${ }^{54}$. Le gouvernement japonais s'intéressa notamment au développement d'une ligne de navigation japonaise sur les côtes caraïbes de la Colombie.

Cette mission d'expertise japonaise souleva le potentiel agraire du pays et le besoin de moderniser les industries urbaines et rurales avec un équipement de bonne qualité et bon marché, ainsi qu'un programme d'immigration japonaise, afin de remédier à la précarité de la main-d'œuvre. Pour les routes montagneuses des Andes colombiens, les industriels nippons conçurent au cours des années 1930 une voiture adaptée à la géographie du pays, la petite Datsun, plus résistante, consommant peu d'essence et d'un prix inférieur d'un tiers à celui de toute voiture nord-américaine. À Buenaventura, une raquette de tennis japonaise se vendait 50 centimes, quatre chemises coûtaient un dollar, le commerce japonais avait de quoi concurrencer les exportations nord-américaines, jugées chères et de mauvaise qualité 55 . Du détroit de Magellan à la Californie, leurs bateaux accaparèrent les pêcheries entre 1920 et 1930, à la grande inquiétude des industries de pêche étasuniennes, et sur la côte caraïbe colombienne, la Kyodo Fishing Company entretint deux antennes fixes ${ }^{56}$. Au Nicaragua, en Colombie et au Salvador, l'importance de l'exportation de café au Japon fut telle entre 1930 et 1935, qu'elle doubla en cinq ans celles des États-Unis ${ }^{57}$. Depuis 1936, occupé par ses conquêtes en Chine, le Japon laissa de côté ses efforts en Amérique du Sud. L'Allemagne, au contraire, intensifia les siens.

En Colombie, l'appel à l'expertise allemande en matière pétrolifère fut abondant et en concurrence directe avec les experts français et nord-américains ${ }^{58}$. En 1921, le Ministère des Travaux Publics engagea l'expertise des géologues allemands Otto Stutzer (1881-1936) et Robert Scheibe (1859-1923), afin d'étudier les concessions pétrolifères sollicitées par les États-Unis, le Royaume-Uni et les Pays-Bas ${ }^{59}$. Robert Scheibe fut particulièrement important pour les études des concessions des mines et des réserves de pétrole dans les régions de Tocaïma, Tolima, Antioquia, Nemocón et Muzo. Bien connu par les autorités colombiennes, M. Scheibe arriva en 1914, étudia et parcourra toute la géographie du pays. Son fils, Ernest Scheibe devint au cours de

53 El Tiempo, año XVIII, n 5904, 2-III-1928, 5.

54 Dépêche $\mathrm{n}^{\circ} 38$ du Ministre de la République en Colombie à M. le Ministre des Affaires Étrangères, 2-III-1928. ADC, 39CPCOM, N 79 .

55 Dépêche n 102 de M. Aymé-Martin, Chargé d'Affaires de France en Colombie à M. S. Pichon, Ministre des Affaires Étrangères, 31-XII-1919. ADC, 14CPCOM, N³.

56 Dépêche $n^{\circ} 75$ de M. de Martel, Ambassadeur de la République française au Japon à M. le Ministre des Affaires Étrangères, 26-VII-1930. ADC, 14CPCOM, N²2.

57 Presseisen, 2013: 42.

58 Dépêche $\mathrm{n}^{\circ} 15$ de M. Langlais, Chargé d'Affaires de France en Colombie à M. Briand, Président du Conseil, Ministre des Affaires Étrangères, 2-III-1922. ADC, Série SdN, Nº 2037.

59 Ibídem. 
1933 un influent expert dans l'exploration des mines de charbon dans la région du Caldas $^{60}$.

Ce fut à ces experts allemands établis en Colombie que 1'Allemagne acheta les minerais, les matières premières et les denrées dont elle avait besoin, en échange des produits métallurgiques pour le développement des réseaux de communication notamment. Cette harmonieuse répartition de l'offre et de la demande favorisa le développement d'un actif flux commercial, qui augmenta à partir de 1936, moment où le régime hitlérien généralisa le principe du troc et mit à la disposition de sa clientèle des marks d'exportation.

Seize accords bilatéraux de coopération économique et technique furent signés par les États d'Amérique latine, depuis l'avènement au pouvoir du NSDAP jusqu'en 1938. En Colombie, la première administration d'Eduardo Santos, signa peu après son élection à la magistrature suprême, un accord avec un groupe de Hambourg pour échanger 700.000 sacs de café contre 800.000 marks payés en charbon et en marks d'exportation. La Société colombo-allemande des transports aériens (SCADTA), fondée en 1919, bénéficia de l'expertise aérienne allemande et des rapports de la Commission internationale de navigation aérienne de la $\mathrm{SdN}$ pour son développement. L'administration d'Eduardo Santos accorda également un premier crédit de 3 millions de dollars pour la commande de 25 locomotives et de 1.000 voitures allemandes, qui devaient remplacer les vieux trains qui dataient de la fin du XIX $^{\mathrm{e}}$ siècle $^{61}$.

La coopération de la Colombie avec les acteurs de la scène internationale est, comme on peut le constater, abondante dans divers domaines. Dans les différentes commissions de la SdN et de l'OIT, ainsi que dans la résolution du conflit colombopéruvien, on retrouve un rapport direct entre le besoin de stabilité sociale et économique à l'intérieur et d'environnement pacifique à l'extérieur. Ces échanges ne furent pas pour autant complètement enthousiastes, car la Colombie commença à considérer le rôle de la $\mathrm{SdN}$ assez limité, préférant plutôt jouer un rôle passif dans les débats des assemblées à partir de $1936^{62}$. En outre, les rapports entre les pays latinoaméricains et les grandes puissances continuèrent à être inégaux malgré l'œuvre de la SdN. Pour la Colombie, l'unité des pays américains à travers le Panaméricanisme pouvait devenir une grande source de profit pour la résolution des conflits au sein des États et placer la région comme un modèle de paix pour les pays européens.

\section{Le projet colombien d'une ligue des nations américaines: renforcer la coopération régionale}

Face aux graves problèmes s'étant posés à la SdN lors de l'occupation italienne de 1'Ethiopie, les chefs d'État américains se prononcèrent majoritairement pour la recherche de nouvelles formes de coopération internationale. En dépit de l'adhésion que la Colombie et d'autres États de la région manifestèrent envers les mesures des organisations genevoises, les républiques latino-américaines membres de la $\mathrm{SdN}$ se virent dans l'obligation de formuler un autre modèle de coopération à partir du régionalisme ${ }^{63}$.

\footnotetext{
Singewald, 1943: 116.

Monthly reports on Colombia, 12-III-1939. AILO, P2589.

Ibídem.

Plata-Stenger, 2015: 99-102.
} 
La Colombie en particulier, vit dans l'aversion chaque jour plus intense des opinions publiques contre la $\mathrm{SdN}$, qu'on retrouve souvent dans la presse latino-américaine, non pas une opposition de l'idéal qu'elle représenta, mais la nécessité de réformer les moyens employés pour atteindre l'idéal de paix et de prospérité mondiale. Les élites latino-américaines multiplièrent au cours des années 1930 des critiques à l'adresse de la SdN. Cette dernière "n'a vécu qu'à travers l'appui des nations américaines" ${ }^{64}$, déclara le président Alfonso López Pumarejo. Il rajouta: “c'est leur présence qui seule, a pu donner à la Ligue son caractère d'universalité" ${ }^{\prime 65}$.

L'historiographie a souligné récemment le rôle joué par l'Amérique latine dans l'internationalisation des organes genevois, mais sans trop mettre en évidence les connexions, les transferts et les refus avec cette région ${ }^{66}$. D'une part, si Genève se montra fidèle aux principes du Convenant, très rapidement elle arrangea les principes et les règles du Droit international aux besoins des grandes puissances, aux besoins des nations européennes et au désir d'éviter à tout prix la guerre. Les nations latino-américaines furent ainsi amenées à accepter des compromis peu avantageux dans l'application intégrale du droit. D'autre part, les grandes nations, durent en raison du contexte de crise, abandonner les règles du droit financier en matière de paiement de dettes par exemple.

Nombreuses furent les initiatives pour réaliser cette réforme: celle d'une ligue des nations américaines formulée par Baltasar Brum en 1923 ; celle du Salvador en 1933 et le projet colombien en 1936 furent le reflet d'un mécontentement croissant des États latino-américains à l'égard de la $\mathrm{SdN}^{67}$. Ces projets eurent pour objectif de doubler, mais à l'échelle régionale l'Institut de Genève et ses organes, entités destinées à être tantôt indépendantes, tantôt rattachées à la SdN, en vertu de l'Article 21 du Pacte de la Société des Nations ${ }^{68}$. Les diplomates colombiens Francisco José Urrutia, Jesús María Yepes (1892-1962) et Jorge Soto del Corral (1904-1955) trouvèrent dans l'histoire latino-américaine et plus précisément sud-américaine, le préambule des organisations de coopération internationale à l'échelle continentale, depuis l'initiative de Simón Bolívar lors du Congrès de Panama : le Panaméricanisme ${ }^{69}$. Analysé par l'historiographie comme un élément complémentaire de la politique étrangère nord-américaine depuis la fin du XIX ${ }^{\mathrm{e}}$ siècle, les conférences panaméricaines furent largement conduites par les États latino-américains durant l'entre-deux-guerres ${ }^{70}$. Le projet présenté par la Colombie et par la République Dominicaine lors de la Conférence de Buenos Aires de 1936 eut pour objectif de créer sur la structure de la SdN une ligue des nations américaines, où tous les États de la région seraient égaux afin d'accorder une importance aux "petites puissances" pour le maintien de la paix universelle $e^{71}$

Concernant sa structure, le projet colombien calquant sur le modèle genevois établit des organes, constitués en principe par un congrès panaméricain, composé de

64 Dépêche n 65 de M. François Gentil, Ministre de France en Uruguay à M. Georges Bonnet, Ministre des Affaires Étrangères, 21-IV-1938. ADC, Série SdN, N 796.

65 Ibídem.

66 Cf. Lespinet-Moret - Viet, 2011.

67 Wehrli, 2008: 18.

68 "Les engagements internationaux, tels que les traités d'arbitrage, et les ententes régionales, comme la doctrine Monroe, qui assurent le maintien de la paix, ne seront pas considérés comme incompatibles avec aucune des dispositions du présent article".

69 Scarfi, 2017: 5.

70 Cf. Vázquez García, 2001.

71 Scarfi, 2017: 82-83. 
trois délégués de chaque nation américaine au maximum, chaque nation ayant une voix. Les nations membres de cette nouvelle ligue suivraient une politique basée sur des principes éthiques: la force ne crée pas le droit et les questions territoriales ne peuvent pas être réglées par la violence. Le président Alfonso López Pumarejo souhaita particulièrement une liberté du trafic des personnes et des biens à travers leurs territoires maritimes, terrestres et aériens. La résolution des conflits régionaux serait dénouée d'après le projet colombien, par une commission régionale d'enquête, une commission de conciliation et d'arbitrage, conformément au Traité d'Arbitrage Panaméricain du 5 janvier 1929, légèrement modifié, et par une cour de justice américaine. Même si ce projet fut présenté comme un élément complémentaire de l'œuvre menée depuis Genève, il reflète une profonde empreinte de la doctrine Monroe. Pour le président colombien, les nations américaines ne devaient pas s'occuper des affaires européennes et réciproquement. L'Europe étant depuis la fin du XIX ${ }^{\mathrm{e}}$ siècle "un continent en pleine décadence, où l'on ne se sert des principes du Droit international que pour couvrir des intérêts et des ambitions ; c'est un continent avec lequel un monde jeune, sain, moral et vigoureux comme l'Amérique doit avoir le moins de contact possible"72. Un article du journal El Tiempo, daté du 15 mars 1938 rapporte:

Ce projet est animé d'une inspiration libérale, pacifique et démocratique. C'est un projet de fraternité des peuples que nous désirons voir se réaliser en Amérique, mais que l'Europe fatiguée et pleine des questions insolubles ne peut réaliser [...] Cette Europe a perdu son prestige parmi les jeunes nations qui rêvent de justice et vivent d'une vraie vie démocratique [...] Si la Ligue des Nations de Genève s'effondre, c'est parce que les nations qui y sont entrées n'ont pensé s'en servir que pour leurs aspirations impérialistes [...] Elle le doit aussi aux erreurs du Traité de Versailles [...] Et aux forces dissolvantes exercées par les États artificiellement crées par la diplomatie et pour la guerre. Notre grand problème commun dans l'avenir est de nous défendre contre l'Europe ${ }^{73}$.

Pour le gouvernement colombien, la $\mathrm{SdN}$ faillit dans son rôle de gardienne du Droit international, et dans le contexte du conflit colombo-péruvien, il aurait aimé voir son application rigide. Dans ces conditions, les États américains, préoccupés de la sauvegarde de la paix et de l'application stricte du Droit international, n'eurent pas d'autres moyens que s'ériger comme "un bloc à haute moralité juridique"74. À partir de 1934, la coopération technique devint le seul trait d'union entre les pays sud-américains et la $\mathrm{SdN}$, en particulier pour le cas colombien, où on retrouve une mobilisation importante de l'expertise genevoise pour l'adoption des lois sociales et des mesures fiscales et financières ${ }^{75}$. Il faut remarquer que lors du conflit de Leticia, la SdN fit appel au concours technique européen et américain, sans nécessairement avoir recours aux membres de la Ligue et le résultat fut positif.

72 Lettre du Ministre de France à Bogotá à M. Yvon Delbos, Député, Ministre des Affaires Étrangères à Paris, 28III-1938. ADC, Série SdN, No 795 .

73 El Tiempo, año XXVII, n ${ }^{\circ} 9505,15-$ III-1938, 3.

74 Dépêche n ${ }^{\circ} 205$ du Chargé d'Affaires de France aux États-Unis à M. le Ministre des Affaires Étrangères, 19-VI1936. ADC, Série SdN, Nº 795.

75 Du côté de l'OIT, on retrouve notamment les missions: Alfredo Vázquez Carrizosa (1934), Moisés Poblete Troncoso (1935), Oswald Stein (1941), Emil Shoenbaum (1945), Marguerite Thibert (1947) et Alejandro Flores Zorilla (1948). 
Alfonso López Pumarejo proclama formellement la faillite de la SdN, devenue un simple "club de discussion", mais en soulignant la nécessité d'une permanente coopération dans le domaine technique entre Genève et l'Amérique latine. Cette dernière devait se placer "comme la gardienne du Droit international le plus pur et strict, en attendant que l'esprit de justice et d'égalité internationale réapparaisse sur les bords du lac de Genève"76. Pour le gouvernement colombien, les pays sudaméricains devaient s'unir autour de l'égalité juridique des États, de l'indépendance des peuples, et condamner la guerre et la politique du plus fort. Le président López fut l'auteur principal dudit projet, soutenu par la République Dominicaine, surtout par le diplomate dominicain Max Henríquez Ureña (1886-1968), ainsi que par son secrétaire au Ministère des Relations Extérieures Alfredo Michelsen et Jorge Soto del Corral, alors ministre de Colombie à Paris et ami intime du président.

La société des nations américaines telle qu'elle a été conçue par la Colombie fut contestée par le Chili, l'Argentine et l'Uruguay, qui manifestèrent leur appui aux organes genevois, en craignant des représailles de la part des régimes allemand et italien, car les immigrants de ces États constituèrent une partie importante de la population du Cône Sud. Le gouvernement chilien en particulier, souligna que la SdN et l'OIT s'occupaient déjà des questions proposées par le gouvernement colombien, tandis que les chancelleries de Cuba, du Nicaragua, du Panama, du Venezuela et de la République Dominicaine manifestèrent un vif intérêt pour l'établissement de cette nouvelle forme de coopération américaine. On pourrait penser que l'intrusion des États-Unis dans les affaires politiques, économiques et militaires en Amérique centrale influença ce choix, car dans la correspondance échangée entre Alfonso López Pumarejo et le président du Panama, Harmodio Arias (1932-1936), on retrouve une volonté de créer un "bloc caribéen" à travers ce projet de coopération régionale, où l'application stricte du Droit international concernant le respect de la souveraineté et la coopération technique seraient les piliers des relations continentales ${ }^{77}$. Les ÉtatsUnis soutinrent la proposition colombo-dominicaine, en soulignant l'inefficacité de Genève vis-à-vis des désordres en Europe et une volonté d'établir aux côtés de la Colombie une nouvelle ère dans les relations des États américains. On peut voir que la Colombie eut un intérêt particulier pour réformer les défaillances des organes genevois à partir de la région latino-américaine, en attribuant à cette dernière un caractère "symbolique", dans un contexte de tension grandissant en Europe et en Asie, sans renoncer pour autant à l'expertise technique genevoise.

Lors de l'arrivée au pouvoir d'Eduardo Santos en 1938, ce projet de coopération américaine se paralysa. Ce fut en raison de l'optimisme de M. Santos envers la SdN, que le gouvernement colombien décida de renoncer au projet de M. López Pumarejo, préférant plutôt renforcer ses liens avec les Etats-Unis autour de la question de la sécurité continentale. La visite à Bogotá de la mission de Luis Podestá Costa en juin 1938, nous révèle que Genève face à la menace du projet d'une ligue des nations américaines, décida d'influencer la nouvelle administration Santos afin de souder davantage ses liens avec la Colombie, au moyen de la coopération intellectuelle ${ }^{78}$. Eduardo Santos fut de plus délégué de la Colombie auprès de la SdN pendant les

76 Dépêche n 237 de Jules Henry, Chargé d’Affaires de France aux États-Unis à M. le Ministre des Affaires Étrangères, 16-VII-1938. ADC, Série SdN, Nº 795.

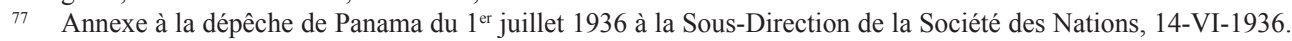
ADC, Série SdN, No 795.

78 Kiddle, 2015: 249-252. 
années 1930, et cela influença sa décision à l'égard de ladite ligue. Ainsi, le décret 2203 de décembre 1938, créa la Commission nationale de coopération intellectuelle en lien direct avec l'Institut international de coopération intellectuelle à Genève. Néanmoins, si l'administration Roosevelt renforça ses liens avec les autres pays latino-américains, ce fut avant tout pour se préparer à un nouveau conflit en Europe. Le sentiment de crainte d'une excessive influence de la Grande République du Nord dans les affaires internes n'avait pas complètement disparu des esprits en Colombie, et la “doctrine Roosevelt" ne suscita pas l'unanimité parmi 1'élite politique ${ }^{79}$.

Quoi qu'il en soit, la politique étrangère colombienne durant l'entre-deux-guerres influença considérablement la recherche de nouveaux mécanismes de coopération régionale et renforça l'attractivité du pays pour les investisseurs étrangers. C'est pourquoi, plus que ses abondantes ressources naturelles et sa diplomatie, ce fut par sa contribution au positionnement des pays latino-américains en tant que "puissances à haute moralité juridique", que Bogotá entendit se faire remarquer des autres acteurs sud-américains ${ }^{80}$. Toutefois, outre l'accroissement du volume de ses échanges avec le reste du monde, l'entrée de la Colombie au nouveau concert mondial après 1919, permit aux dirigeants colombiens le renforcement du système oligarchique sans pour autant résoudre efficacement les graves problèmes sociaux qui s'accentuèrent durant cette période.

\section{Conclusion}

Dans un monde marqué par les échanges et les transferts de personnes, d'idées et d'objets, l'organisation du système international s'est considérablement développée durant l'entre-deux-guerres. Dans ce contexte, la politique étrangère colombienne chercha par tous les moyens le renforcement de la coopération régionale et internationale pour éviter le surgissement du désordre social, incompatible, d'après les administrations colombiennes, avec les principes humanistes et le développement économique. L'intégration de la Colombie au système international issu du Traité de Versailles tenait à sauvegarder les principes du Droit international et ouvrir un débat sur le rôle de l'Amérique latine dans la recherche de la paix et le développement du monde. On peut voir dans la formulation de cette politique étrangère un certain nombre d'avantages pour le développement du pays. La diversification de ses relations avec les autres acteurs de la scène internationale lui permit d'obtenir un prestige moral, motivant un accroissement des investissements étrangers et la recherche d'autres modèles de développement matériel, social, politique et culturel dont la Colombie eut besoin pour répondre efficacement aux rapides transformations de sa société, de son économie et les défis que cela représenta pour ses institutions.

\section{Bibliographie}

Allain, Jean-Claude. "La moyenne puissance: une réalité fuyante de la vie internationale". En Enjeux et puissances, pour une histoire des relations internationales au XXe siècle.

79 Randall, 1992: 176

80 Urrutia, 1920: 154. 
En Mélanges en l'honneur de Jean-Baptiste Duroselle, édité par Duroselle, Jean-Baptiste - Institut d'Histoire des Relations Internationales Contemporaines. Paris: Publications de la Sorbonne, 1986, 11-27.

Bureau international du travail. Conférence internationale du travail. Première session annuelle 29 octobre - 29 novembre 1919. Genève: Sonor, 1919.

Castillo, Antonio del. Antecedentes del Panamericanismo, Panamá, del Congreso de 1826 a la reunión de Presidentes Americanos en 1956. Bogotá: Editorial Iqueima, 1956.

Conférence internationale du travail. Conférence internationale du travail. Douzième session. Genève: Sonor, 1929.

Economic and Financial Section of the League of Nations. International Statistical YearBook. Genève: Publications of the League of Nations, 1927.

Frank, Robert. Pour l'histoire des relations internationales. Paris: PUF, 2012.

Gamboa Ardila, Néstor, Raúl. La economía colombiana: del modelo de protección al modelo de apertura [2005]. Medellín: Universidad de Medellín, 2007.

Gilhodes, Pierre. "La politique extérieure de la Colombie". En La Colombie à l'aube du troisième millénaire, coordonné par Blanquer, Jean-Michel - Gros, Christian. Paris: Éditions l'IHEAL, 1996, 123-136.

Henderson, James. Modernization in Colombia: The Laureano Gómez Years, 1889-1965. Gainesville: University Press of Florida, 2001.

Herrera León, Fabián - Wehrli, Yannick. "Le BIT et l'Amérique latine durant l'entre-deuxguerres: problèmes et enjeux". En L'Organisation internationale du travail, origine, développement, avenir, coordonné par Lespinet-Moret, Isabelle - Viet, Vincent. Rennes: Presses Universitaires de Rennes, 2011.

Kiddle, Amelia. "Separating the Political from the Technical: The 1938 League of Nations Mission to Latin America". En Beyond Geopolitics: New Histories of Latin America at the League of Nations, édité par Mcpherson, Alan - Wehrli, Yannick. Albuquerque: University of New Mexico Press, 2015, 239-257.

Lespinet-Moret, Isabelle - Viet, Vincent (dirs). L'Organisation internationale du travail, origine, développement, avenir. Rennes: Presses Universitaires de Rennes, 2011.

Plata-Stenger, Véronique. "To Raise Awareness of Difficulties and to Assert Their Opinion: The international Labour Office and the Regionalization of International Cooperation in the 1930s". En Beyond Geopolitics: New Histories of Latin America at the League of Nations, édité par Mcpherson, Alan - Wehrli, Yannick. Albuquerque: University of New Mexico Press, 2015, 97-113.

Presseisen, Ernst. Germany and Japan: A Study in Totalitarian Diplomacy 1931-1941. La Haye: Springer, 2013.

Randall, Stephen. Colombia and the United States: Hegemony and Interdependence. Athens - London: The University of Georgia Press, 1992.

Rausch, Jane. Colombia and World War I: The Experience of a Neutral Latin American Nation during the Great War and Its Aftermath, 1914-1921. Maryland: Lexington Books, 2014.

Rivas, Raimundo. Historia diplomática de Colombia, 1810-1934. Bogotá: Imprenta Nacional, 1961.

Safford, Frank. El Ideal de lo práctico. El desafio de formar una élite técnica y empresarial en Colombia. Bogotá: Universidad Nacional-El Ancora Editores, 1989.

Sánchez, Gonzalo. Guerre et politique en Colombie. Paris: L'Harmattan, 1998.

Scarfi, Juan Pablo. The Hidden History of International Law in the Americas, Empire and Legal networks. Oxford: Oxford University Press, 2017. 
Singewald, Joseph. Bibliography of Economic Geology of South America. Maryland: Geological Society of America, 1943.

Urrutia, Francisco José. La evolución del principio de arbitraje en América. La Sociedad de las Naciones. Madrid: Editorial-América, 1920.

Vázquez Carrizosa, Alfredo. Relatos de historia diplomática de Colombia, siglo XX. t. I. Bogotá: Ministerio de Relaciones Exteriores y Centro Editorial Javeriano, 2000.

Vázquez García, Humberto. De Chapultepec a la OEA: Apogeo y Crisis del Panamericanismo. La Habana: Ciencias Sociales, 2001.

Wehrli, Yannick. "Los proyectos de 'Sociedad de las Naciones Americanas': intentos de integración política en las Américas durante el periodo de entre-guerras". En Los procesos de integración en el ámbito regional y global. Una mirada desde la perspectiva de los tres continentes (América, Asia, Europa), édité par Monserrat Llairó, María de - Palacio, Proscila. Buenos Aires: CEINLADI-FCE-UBA, 2008, 1-17. 